CASOS CLINICOS

Rev. Chil. Pediatr. 68 (2); 93-98, 1997

\title{
Trisomía 8: reporte de cuatro casos
}

\author{
Cecilia Mellado S. ${ }^{\text {; }}$ Rodrigo Moreno S. ${ }^{2}$; Fermina López G. ${ }^{3}$; \\ Patricia Sanz C. ${ }^{4}$; Silvia Castillo T. ${ }^{4}$; Cecilia Villaseca G. ${ }^{1}$; Vera Daher N. ${ }^{5}$; \\ Lorena Tobella P. ${ }^{3}$; Samuel Salazar C. $^{5}$
}

\begin{abstract}
Resumen
La sisomía 8 es una anomalía cromosómıco que en la mayoría de los casos descritos corresponde a un mosaico Sus coracteristicas clínicas vorían desde dismorfias discretos hasta malformaciones severas que. por lo general, incluyen retardo mental feve a grove-, dismorfios faciales típicas, alteraciones esquelélicas, pliegues palmares y plartares profundos, anomalías renales y otras Con el propósito de ilustrar la variedod de los coracterísticas tenorípicos de estos onomalias se describen los casos clínicos de cuatro pacientes cuyo diagnóstico se confirmó citogeneticamente, tres con trisomía en mosaico y uno con trisomio 8 completo. La solicitud del estudio citogenérico Iuvc su origen en la dismortia, retroso del desarrollo psicomotor o def lenguoje o hipotonía muscular esquelériça. Es itrportante rener en cuenta la voriedad de las característicos tenotipicas de esta trisomia, para sospechaí correctamenle el diagnóstico y solicitar oportunamente el estudio cílogenético.
\end{abstract}

(Palabras claves trisomía 8, variación tenotípica, moscicismo.)

\section{Chromosome 8 trisomy}

Trisomy 8 is a chromosomal disorder occurrig usually as a mosaicism. The clinical charocteristics of affected patienrs are quite variable ranging from miłd ic severe mental rerardation and dysmorphic Features, including focial malformations, skeletal abnormalities, deep palmor and plantar creases and rencl anomalies among most relevant phenotypic facts. To illustrate this differe-t clinicol polterns four cases of trisomy 8 are reported, three of them were mosaics and one was a full risomy. Patients were referred to sludy by dismorphic lacies, delayed achievement of developmental milestones or speech or hypatonio. Phenolypic variability of this synorome must be token into account in the diagnostic approoch of obnormality.

[Key words: trisomy 8, mosoicism.

En 1963 se describió por primera vez un síndrome causado por trisomía $C^{\prime}$; ocho años más tarde De Grouchy identificó al cromosoma 8 como responsable del trastorno. gracias al de-

I. Médicos del programa de formación de especialistas en Genética Clinica, Servicio de Genética, Departamento de Medicina Hospital Clínico de la Universidad de Chile.

2. Servicio de Genética, Hospital Exequiel González Corlés

3. Servicio de Genética, Hospital Gustavo Fricke, Viña del Mar.

4. Servicio de Genética, Departamento de Medicina, Hospital Clínico de la Universidad de Chile.

5. Tecnólogos Médicos Servicio de Genética, Departamento de Medicina, Hospital Clínico de la Universidad de Chile. sarrollo de los métodos que permiten el revelado de bandas cromosámicas (bandeo) ${ }^{2}$. El primer caso fue descrito en Chile el año $1986^{3}$.

La trisomía 8 es relativamente poco frecuente en el hombre, ocurre en aproximadamente 1,2 por mil de todos los embarazos reconocidos clínicamente y 8 por mil los abortos espontáneos ${ }^{4}$. Su frecuencia se estima entre 1 por 25000 y 1 por 50000 niños, con una razón masculino/femenino de $5: 1^{5}$. Su expresión fenotípica es variable. pues algunos individuos sufren dismorfias leves -por lo que en algunos pacientes no se hace el diagnóstico-, mientras otros son intensamente afectados.

El diagnóstico de trisomía 8 se basa en la presencia de un cromosoma 8 adicional, habj- 
Lualmente en mosaico y, generalmente, con una línea celular normal 1. 2, 5-10. La distribución del cromosoma adicional es también variable de uno a otro paciente en los distintos tejidos. En algunos la alteración se encuentra sólo en fibroblastos, en otros puede predominar en los linfocitos y no registrarse en otros tejidos o aparecer en sólo una pequeña proporción de las céJulas correspondientes. En algunos casos de Irisomía completa en linfocitos, en los cuales se examinaron tejidos adicionales, se encontró usualmente mosajeismo en al menos uno de los tejidos examinados ${ }^{\prime \prime}$. Parece no haber relación entre la proporción de células trisómicas y la severidad de las manifestaciones clínicas ${ }^{12,}$,3. Los casos de trisomía 8 completa son raros y generalmente incompatibies con la vida, terminando en abortos espontáneos en el período temprano del desarrollo embrionario ${ }^{14}$. La trisomáa 8 es también un hallazgo frecuente en los cromosomas de la médula ósea de pacientes con distintos desórdenes hematológicos y se han descrito enfermedades malignas en $4 \%$ de 10 s pacientes con la aneuploidía, pero el significado de este hecho se desconoce. 6.

Esta trisomía ocurre de novo. No se han identificado padres portadores. Su causa puede ser una no disyunción meiótica (precigótica) con pérdida parcial postcigótica del cromosoma 8 cxtra, o una no disyunción mitótica (postcigótica). Al parecer es más frecuente la no disyunción mitótica, lo que explicaría el mosaicismo, la larga sobrevida y el buen pronóstico de estos pacientes $^{9-12}$.

A continuación se describen cuatro pacientes con trisomía 8 para destacar sus características clinicas más importantes. con el fin de subrayar la conveniencia de tener presente la variedad de formas de presentación que puede exhibir esta anomalia, sospecharla y realizar el estudio cromosómico correspondiente. El análisis citogenético de todos los pacientes fue realizado en preparaciones oblenidas de cultivos de rutina de $72 \mathrm{~h}$ de linfocitos de sangre periférico, teñidos con el método de bandeo $G$ convencional. No se estudiaron células de otros tejitos.

\section{Casos clínicos}

1. Varón nacido el 4 de febrero de 1991. La madre de 28 jŏos dueña de casá. tenía una hija previa sana. El padre, de
22 años de edad, obrero en una fábrica de cemento, fumaba un tigarrillo diario y bebia alcohol ocasionalmente. Sin an. tecedentes familiares de consanguinidad, malformaciones, retardo mental o abortos. El embarazo cursó con colestasia intrahepática (CIE) desde el quinto mes. Parto vaginal de término. fue inducido a causa de la CIE y requirió aplicación de fórceps por distocia de posicjón. Al nacer pesabia $3240 \mathrm{~g}$, medía $50 \mathrm{~cm}$ y su circunferencia craneana era de $35 \mathrm{~cm}$. Por hiperbilirrubinemia neonatal se mantuvo en el hospital en fototerapia. Después del período reonatal el paciente evoluciond con problemas respiratorios repetidos, retardo global del desarrollo psicomotor y del lenguaje y a la edad de 4 años sufrio dos episodios de convulsiones. A la edad de $S$ arios 5 meses pesaba $17 \mathrm{~kg}$. media $103 \mathrm{~cm}$, su perímetro craneano era de $54 \mathrm{~cm}$. Braquicefalia. Orejas de hélix plegado. antihélix prominente, rotadas hacia atrás. Ojos profundos, fisuras horizontales y ptosis palpebral. Nariz de base ancha, narinas antevertidas, filtrum pmominente. Labio superior e inferior gruesos, inferior evertido. Micrognatia leve. Cuello corto, ancho y alado. Hombros angostos. tronco largo en relación a las exiremidades. pecfum excavarum, areolas mamarias hipoplásicas y separadas. Cicatriz de herniorrafia inguinal izquierda e hidrocele operado a derecha. Gevitales masculinos, testículos en el escroto. Surcos palmares profundos, clinodactilia y pliegue únice en el quibto dedo de ambas manos. Surcos planiares profundos, hallux valgus, camptodactilia de anbos pies con clinodactilia de los segundos terceros y cuartos ortejos $y$ del quinto en el pie derecho.

La tomografía axial computadorizada del encéfalo se registró agenesia de cuerpo calloso. En las radiografías de huesos largos había signos de doble cortical y, en los pies. signos de metatarso varo y hallux valgus. Electroencefalograma, escudio metabólico, TSH, T4 y radiografías de columna cervical normales. Cariotipo: trisomía 8 en mosaico con 28 células trisómicas y dos normales $(47, \mathrm{XY},+8(28) /$ $46, X Y(2)$.

2. Varón nacido el 4 de eneto de 1995, segundo hijo de padres sanos, no consanguíneos. Padre de 31 ăhos, recuperador de desechos gráficos, fumaba 10 cigarrillos diarios y consumía alcohoJ ocasionalmente. La madre de 26 a ouos, auxiliar de enfermería, sana. Un hermano mayor sano. EI embarazo cursó sin incidentes. Rotura prematura de membranas y cesárea al $B^{\circ}$ mes de gestación. Peso de nacimien. to $2800 \mathrm{~g}$, talla $49 \mathrm{~cm}$, apgar 8 . Hiperbilinubinemia. hipoglicenia e hipocalcemia neonatales precoces. Posteriomente sufrió reiterados irastornos respiratorios. retardo del desarrolto psicomotor e hipotonfe. A los 12 meses de edad se le realizó orquidopexic. A la edad de 1 año 6 meses pesaba $10500 \mathrm{~g}$, medía $81 \mathrm{~cm}$ de talla, su circunferencia craneana era $48 \mathrm{~cm}$. Hipotonía leve, bien conectado, activo, balbuceaba algunas palabras. Ojos profundos de inclinación mongoloide, con epicanto. Orejas pequeñas. Nariz grande, bulbosa, base ancha, narinas antevertidas. Paladar alto. labios gruesos, el inferior evertido. Cuello forto. Hombros angostos, tronco largo en relación a las extremidades, aréolas manatias hipoplásicas, separadas (figura I). Genitales misculinos, ambos testículos en el escroto. Surcos profundos, clinodactilia del segundo dedo derecho y el quinto izquierdo, camptodactilia del cuarto dedo en las manos. Surcos plantares profundos, cabalgarniento del quinto ortejo sobre el cuarto (figura 2). 

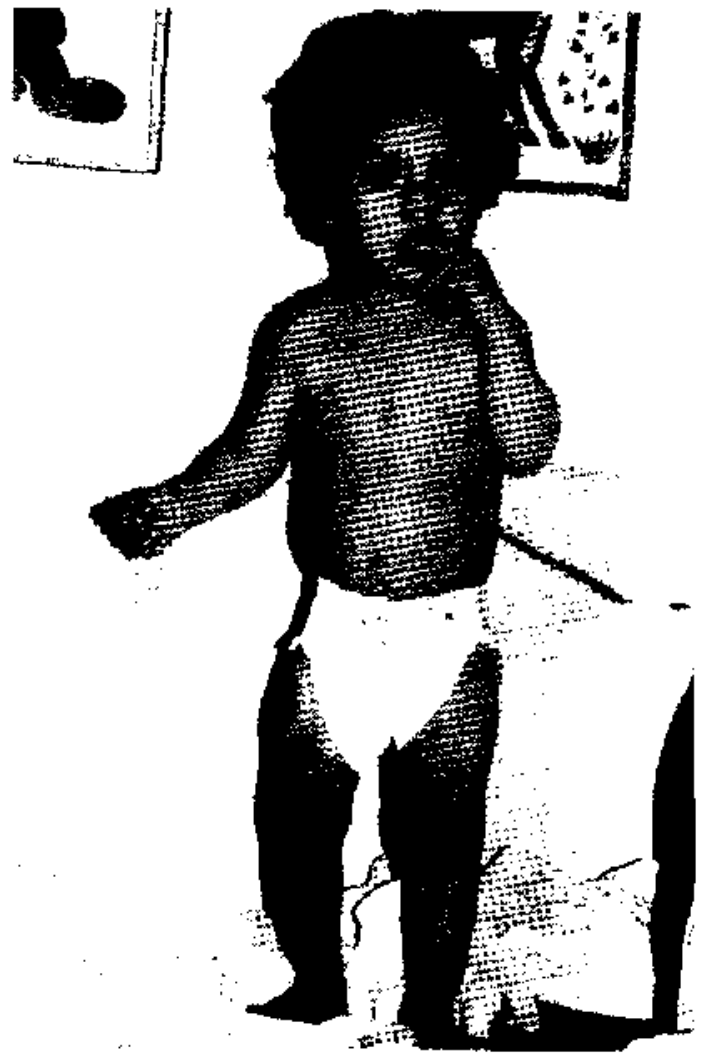

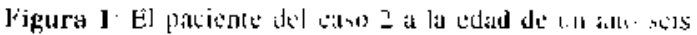

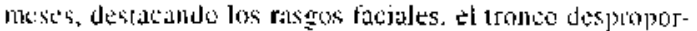
cionadanostc largo y la hipertelia.

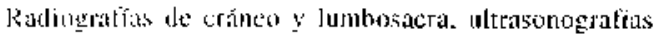

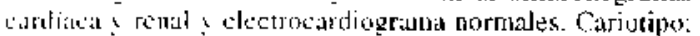

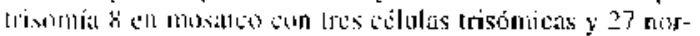

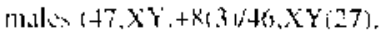

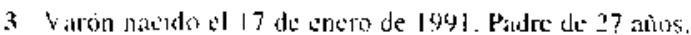
madre $\mathrm{J}$ 23. to consanglimes. Dos hemmonos sanos. Elkb.trize normul. Parto a las 38 semanas de gestasuón. Peso de rasimiconto 3120 g. talla 51.5 inh cireunferencia ira-

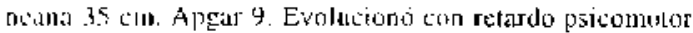

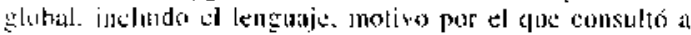
lial edad de 3 ands 2 meses. Pesaha 14950 g. Lalla 96 cor.

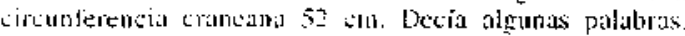
Freme amplia y prommente orejas de antihćlix plegado. Fistrabismo de ojo dertoho. Vare de base ancha, narinatio anteveruidas. Labios gruestsi, s] iuferlis esertido. Cvula bifida. Plieguts palmares y pliutares prulundos. Ortejos miloces en mactillo Aminoriciduria, aminuacidemia. mucupolisacáridos, romografía axial compucindorizadal de cerebro y audiontetría normaks. Carjeripo: Irisomía 8 en

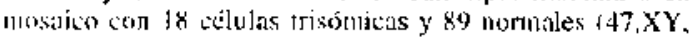
$+\$(13)+46, X Y(89)$.

4. Varon nacido t 19 de octubre de 1993. Primer hijo. Padec de 25 aj̀o, madle de 22 años, sanos, no consangú-

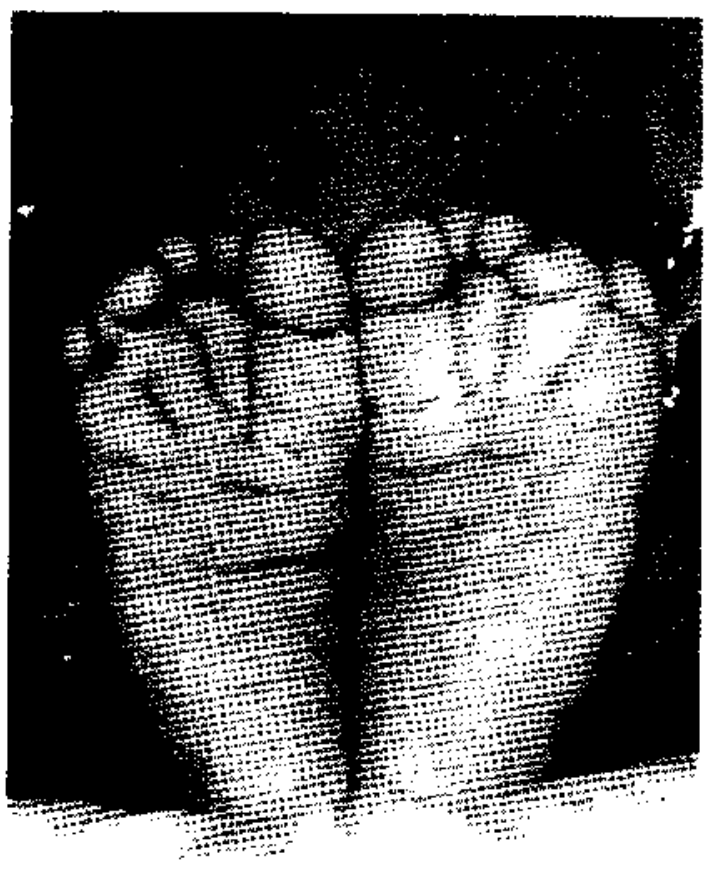

Figura 2: Plicgues plantares profundos y cabaliganiemo do los oitejos.

neos sin antecentes de consumo de cigarrillos, alcohol ni drogas. Embarazo normal. Palto de Iérmino por cesátea por sufrimiento fetal. Pesó 3650 g y midió 54 cull. curcunferencia craneatna $3.5 \mathrm{~cm}$. Apgar 9 En el período nconatal presentó signos de insuficiencia círculatorì. ingresando a la unidad de cuidados intensivos neonatales de su localidad. En cl cráneo mostraba depresion leve frontal biluteral. Ortjas de hêlix hipoplásico, implantáción baja y fosetas preauriculares al hodo dercho. Ofos profundos con hendedurus malpetrales inclinades hacia arriba $y$ afucra. Nariz ancho de punti bulbosa. Bota grande, paladar alto: micrognalta. Tórix largo en relación al resto del cuerpo. areolas mamarias separadas. Sopto carcliaco. Genilales masculinos nornales Superposición del dedo indice dere. cho sohre el terecr dedo: segunda falange de los terceros dedos en flexión fija. Surcos palınares protundos. Ortejos mayores largos de pulpejos anchos, clinudactilia y surtos Jongitudinales profundos udue cruzan los dos tercios anteritres de ambas plantas. Sufrió reileradas infecciones respiracrias hastal la edad de cuatro meses $y$ tres semanas. cuando reingress at hospital por bonconeumonia, anemia. insuficiencia cardíaca congestiva y falleció.

En la ultrasonografía cardíaca se enconsaroll signos de una comunicación interauricular Jel tipo artiun secuadomt 
de to ma de diánelan, comunicución interventricular subatistieta de 2 mun è bipertemilon arterial pulmondr. Las uläavomogratias abolominal y cerebcal dieron resultadom

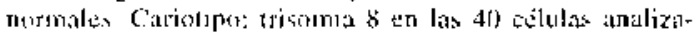

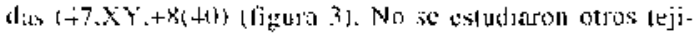

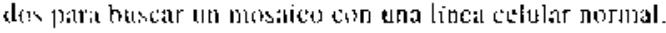

\section{Comentario}

La trisumía 8 licne dilerentes expresiones

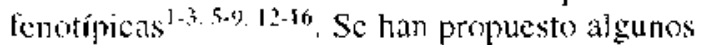
signos lísicos com el propósito de tener presente la aleceión y ofrecer una guía para la investigacion clíntca conducente al diagnóstico $0^{\%}{ }^{9}$. En re(ien nacidas y lactantes se debe suspechar la anomalia ante algunas malformaciones craneolikiales. sobre ado nasolabiales. junto a clinodactilia $y$ pliegues palmares y plantares protundos. Sin embargo, como estos pacientes tienen un huen crecimiento en peso y estatura y sus mallomaciones son leves, por lo general no se hace estudio citogenćtico en esa ctapa de la vidis. En la niñes los plicgues profundos pueden desaparecer. pero se expresan atros signos. co- mo tronce angosto, pelvis estrecha, tendencia a lis rigides articular (camptodactilia) y retardo psicomotor, especialmente del lenguaje".

En la tabla se resumen las características más importantes de la trisomía 8, con las manilestaciones presentes en nuestros pacientes. Los pacientes afectados pueden sufris complicaciones derivadas de sus anomalías esqueléticas o viscerales. comn insuficiencia renal, prohlemas cardioyasculares, restricción de la movilidad articular y retardo psicomotor. El retraso del lenguaje y los problemas articulares son frecuentes, por lo que, una vez hecho el diagnóstico de trisomía 8. estas alteraciones deben tencrse en cuenta para su adecuado mancjo ${ }^{6}$.

Citogenéticaunente, la mayoria de los casos de trisomía 8 descritos son en mosaicol, 3-11. En tres de nuestros casos se comprobaron dos líneas celulares, y sólo en el cuarto se encontro la ancuploidía en todas las células analizadas, pero como en este último no se estudiaron otros tejidos, no es posible descartar mosaicismo. La difercntc proporción de células trisómicas en cada paciente, y grados no concordantes de expresión
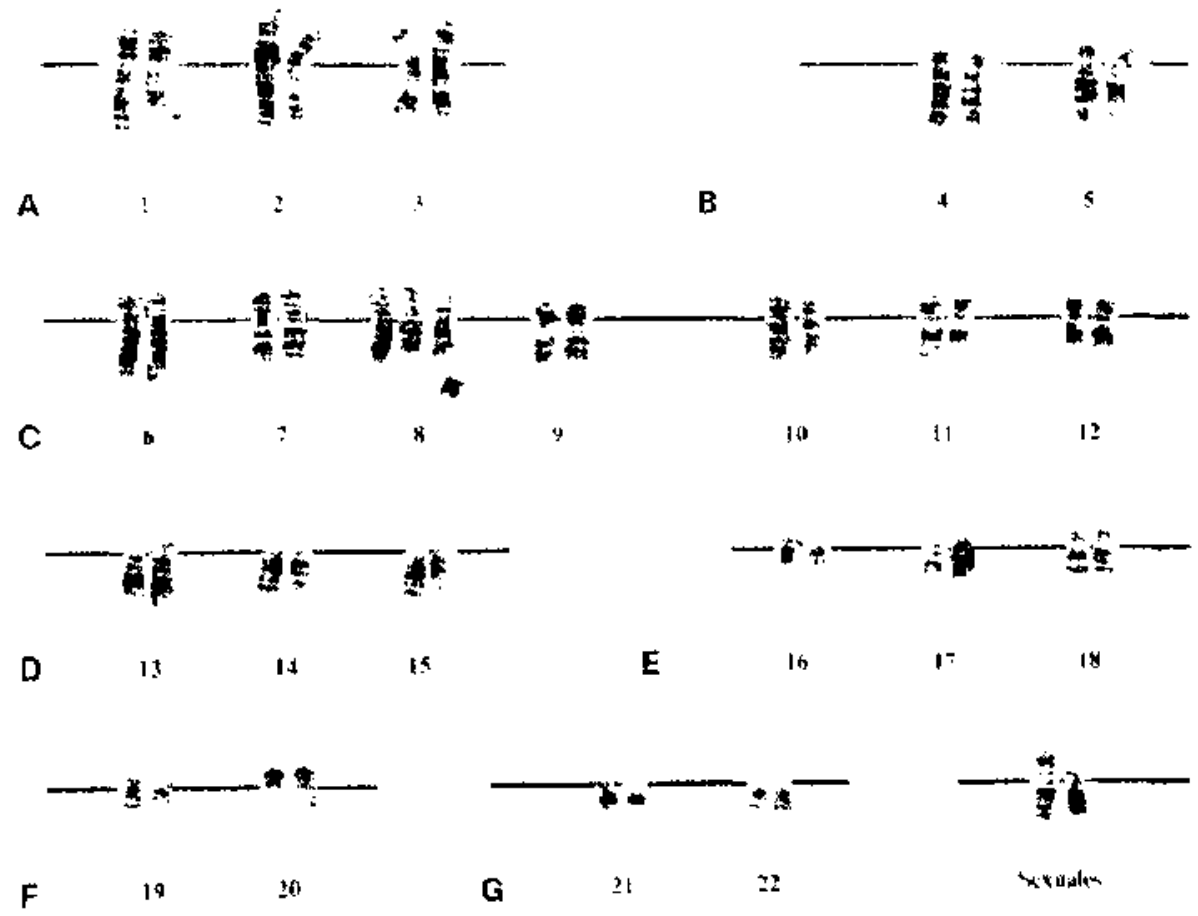

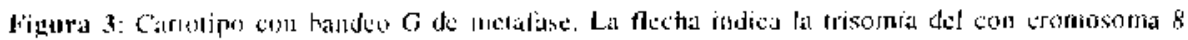
$147, X Y,+8$ s. 
Tabla

Características más frecuentes de la trisomia 8 y las encontradas en los pacientes

\begin{tabular}{|c|c|c|c|c|c|}
\hline Características & & Caso 1 & Caso 2 & Caso 3 & Caso 4 \\
\hline Edad de dingnóstico & De RN (2/3) a adultos (1/3) & $55 / 12 a$ & $5 / 12 a$ & $32 / / 2 a$ & 2 dlas \\
\hline Peso de nacimiento & $3700 \mathrm{~g}(\mathrm{x})$ & 3240 & 2800 & 3120 & 3650 \\
\hline Edad madre/padre & $29 / 33$ afios $(\bar{x})$ & $23-21$ & $24-30$ & $22-27$ & $26-25$ \\
\hline Cariotipo & Mosaico. Trisonía completa infrecuente & Mosaico & Mosaico & Mosaico & Completa \\
\hline \multirow[t]{6}{*}{ Caheza } & Cráneo grande, frente prominente/cara alargada & + & + & + & + \\
\hline & Anomalias de las orejas & + & + & + & \\
\hline & Ojos profundos & + & + & + & + \\
\hline & Anomalías palpebrables & + & + & & \\
\hline & Nariz ancha, narinas antevertidas & + & + & + & + \\
\hline & Labio inferior evertido & + & + & + & + \\
\hline Cuello & Corto o alado & + & + & & \\
\hline \multirow[t]{2}{*}{ Tórax } & Largo, hombros angostos & + & + & & + \\
\hline & Costillas supernumerarias & & & & \\
\hline Columna & Escoliosis, vertebras acces orias/deformes & & & & \\
\hline Pelvis & Angosta & + & + & & \\
\hline \multirow[t]{3}{*}{ Extremidades } & $\begin{array}{l}\text { Clinodactilia/braquidactilia/hallux valgus } \\
\text { Hipopiasia o ausencia rótulas }\end{array}$ & + & + & + & + \\
\hline & Función articular limitada & + & + & & + \\
\hline & Hipotonía & & + & & \\
\hline Cardjovascular & Cardiopatia congénita & & & & + \\
\hline Gastrointestinal & Hernia ingเsinal & + & & & \\
\hline SNC & Agenesia cuerpo calloso & + & & & \\
\hline Urogenital & $\begin{array}{l}\text { Hipospadias /criptorquidia } \\
\text { Anomalias del tracto urinario }\end{array}$ & + & & & \\
\hline \multirow[t]{2}{*}{ Desarrollo psicomotor } & Retraso global & + & + & + & \\
\hline & Retraso del lenguaje & + & & + & \\
\hline Dermatoglifos & Pliegues palmares y plantares profundos & + & + & + & + \\
\hline
\end{tabular}

fenotípica con dicha distribución, ha sido descrita. Las comparaciones entre la proporción de células aneuploides y la magnitud de las manifestacfones clínicas tampoco han mostrado relación entre ellas $7,8,12,13$. La variedad de fenotipos en esta trisomía podría depender de la contribución de las células aneuploides en el desarrollo de los diferentes tejidos ${ }^{7.8}$. Puesto que en estos pacientes la proporción de células trisómicas en los linfocitos disminuye con la edad $2.6 .9 .1+16$, se ha propuesto realizar lo más temprano posible el estudio citogenético o hacerlo en fibroblastos de piel, cuyas células son más estables, si bien se han descrito casos donde no se ha encontrado la aneuploidía en los linfocitos pero sí en los fibroblastos ${ }^{6.9}$.

Es importante tener presentes las diferentes manifestaciones clínicas de la trisomía 8 para sospecharla. solicitar el estudio citogenético y aclarar el diagnóstico en los pacientes que presentan características sugerentes, ya sea en forma severa o leve.

\section{Agradecimientos}

Al Dr. Ronald Youlton R., por su valiosa ayuda en la revisión de este artículo.

\section{Referencias}

1. Jones $K$ : Trisony 8 syndrome. In: Smith's Recognizable Pattems of Human Malformation, edit 4. Philadelphia: W.B. Saunders, 1988: 26-29.

2. De Grouchy J. Turleau C: 8 trisomy. In: Clínical atlas of human chromosomes, ed 2. New York: John Whiley \& sons $1984 ; 126-133$.

3. Morizon G, Aspillaga M: Trisomía 8 mosaico: $46, \mathrm{XX} /$ 47.XX+8. Rev Chil Pediatr 1986: 57: 545-549. 
4. Hassold TJ, Jacobs PA: Trisomy in man. Annu Rev Genet 1984; 18: 69-97.

5. Gorlin RJ, Cohen MM. Levin LS: Syndromes of the head and neck, ed 3. New York: Oxford University Press $1990: 49-50$.

6. Rosengren S, Cassidy S: Chromosome 8, Trisony 8. En: Birth Defects Encyciopedia Dover: Blackweil Scientfic Publications 1990; 351-352.

7. Bery AC, Mutun DE, Lewis DGM: Mosaicism and the trisomy 8 syrdrome. Clin Genet 1978 ; 14: 105. 114.

8. Kumba Z. Krakwa B. Piatkowska E. er ul: Trisomy 8 mosaictisn synúrone. Clin Pediatr 1988, 27: 557-564.

9. Kosztolinyi $G$, Bühler EM, Elminger $P$. et ul: Trisomy 8 mosaicism. Europ I Pediatr 1976; 123: 293-300.

10. Rowend S, Jacobs P: Molecular studies of aetiology of trisomy 8 in spontaneous abortions and the liveborn population. Hum Genet 1996; 97 : 283-286.
11. Robinson WP. Binkert F, Bernasconi F. et at: Molecular studies of chroınosomal mosaicism: relative frecuency of chromosome gain or loss and possible role of cell selection. Ain J Hum Genet 1995: 56: 444451

12. Riccardi VM: Trisomy 8: an international study of 70 patients. Birth Defects 1977: 13: 17i-184.

13. Chatdley AC. Hargreave TB. Fletcher SM, et al: Trisomy 8: report of a mosaic human male with nearnormal phenotype and normal IQ, ascertained trough infertility. Hom Genet 1980; 55; 31-38.

14. Buttros.s S, Massingale T, Feingold M: Trisomy \&/ Mosaic syndrome. AJDC 1990; 144: 507-508.

15. Reyes $P$, Hse L. Strauss L. et al: Trisomy 8 mosaicism syndrome. Report of monozygotic twiss. Clin Genet 1978: 14: $90-97$

16. Rodewald $A$, Zankl H. Wischerath, et al: Dermatoglyphic pattems in trisomy 8 syndrome. Clin Genet 1977: 12: 28-38.

\section{AVISO A LOS AUTORES}

Con el objeto de dar prioridad a los trabajos de investigación, en vista de las limitaciones de espacio de la Revista Chilena de Pediatría, el Comité Editorial ha acordado restringir la impresión de casos clínicos a un máximo de dos por cada número. 